\title{
Ferromagnetic Resonance Measurement Using a Novel Short Circuited Coaxial Probe Technique
}

\author{
Tejinder Kaur ${ }^{1}$, Murthy Devata Venkata Bhyrava ${ }^{2}$, Jose Luis Olvera-Cervantes ${ }^{2}$, and \\ Alonso Corona-Chavez ${ }^{2 *}$
}

\author{
${ }^{1}$ División de Ingenierias - Universidad de Guanajuato, Campus Irapuato-Salamanca, Mexico \\ ${ }^{2}$ Depto. Electronica, Instituto Nacional de Astrofísica, Optica y Electronica, Puebla Mexico \\ *corresponding author, E-mail: alonsocorona@inaoep.mx
}

\begin{abstract}
A versatile technique to characterize the ferromagnetic resonance (FMR) of ferrite samples using a short circuited coaxial probe is presented. The technique has sensitivity comparable to that of well-established methods besides its non-contact nature, broadband and local. Detailed theoretical approach and simulation studies (Proof of Concept) using HFSS are presented. Microwave measurements on different single crystal and polycrystalline samples (Yttrium Iron Garnet $\left(\mathrm{Y}_{3} \mathrm{Fe} 5 \mathrm{O}_{12}\right)$ YIG and Nickel Ferrite $\left(\mathrm{NiFe}_{2} \mathrm{O}_{4}\right)$ NFO have been performed. We measured the FMR response of these samples as a function of frequency and the data showed the expected variation for both in plane and out of plane magnetic fields.
\end{abstract}

\section{Introduction}

The great diversity of ferrites permits coverage over a wide range of frequencies (from $\mathrm{kHz}$ to tens of $\mathrm{GHz}$ ) for a set of appropriate properties. Microwave ferrites allow the control of microwave propagation by a static or switchable dc magnetic field. The devices can be reciprocal or nonreciprocal, linear or nonlinear, and their development requires knowledge of magnetic materials, electromagnetic theory, and microwave circuit theory. The behavior of all microwave ferrite devices can be explained in terms of Faraday rotation and ferromagnetic resonance (FMR). Ferromagnetic resonance (FMR) based ferrite devices are attractive due to high-Q, low-insertion loss, reconfigurability and high out - of band rejection. For most of the applications, the choice was settled on yttrium garnet (YIG), which exhibits suitable properties for microwave components.
Traditionally, FMR is measured using microwave cavities or strip lines, [1-3] where the sample size and geometry are constrained by the measurement apparatus. Resonant structures (coaxial, dielectric, and cavity resonators) are most commonly used to characterize materials at microwave frequencies. A few techniques based on scanning probe microscopy (SPM) for imaging spatial distributions of magnetic response in a range of radio frequency (RF), such as electron paramagnetic resonance and FMR, have been developed [4-7]. FMR signals on a polycrystalline YIG disk has been observed using the openended coaxial electric probe by Toshu et.al [8]. A shorted coax microwave probe is used to explain the near field magnetic microscopy phenomena by Lee et al [8]. The probe was designed by soldering the inner and outer conductors of the coax, resulting in a probe that can easily couple the RF field magnetic field to the sample. This technique is further extended using a thin wire bond to short the coax by Mircea et al [10]. This in turn allowed the use of simpler electronics for the measurements. Recently microwave resonators based on planar ferrite-piezoelectric structures tuned by magnetic and electric fields have been studied [11, 12]. The electric field induced ferromagnetic shift of $\mathrm{BiFeO}_{3}-\mathrm{NiFe}_{2} \mathrm{O}_{4}$ (BFO-NFO) nanocomposites using the shorted micro coax probe explained by Nadjib et al [13]. In this report, we present the compact measurement methodology for the broadband detection of Ferromagnetic Resonance using Short Circuit Coaxial Probe Method. The introduced method is a promising tool to characterize the ferromagnetic resonance frequency, resonant line widths of magnetic materials with high sensitivity and fast testing. We explained in detail, the theoretical support, design, simulation and microwave measurements of the proposed method on different magnetic materials. With this approach, we have successfully detected localized FMR signals on different magnetic samples (YIG single crystal thin film samples, polycrystalline yttrium iron garnet (YIG) and polycrystalline Nickel Ferrite samples) at different frequencies. While the samples studied in this experiment is relatively large with millimeter-size dimensions, limited by the wavelength of the excited magneto static waves. 


\section{Theory}

Here we demonstrate a high-band-width FMR probe, where there is no constraint on the sample size or geometry, thus enabling high-frequency measurements on a multitude of materials in their actual operating conditions. A scanning coax microwave probe with magnetic sensitivity was demonstrated by Lee et al. [9] The probe excites a small sample area and picks up the electromagnetic response using a coaxial transmission line resonator terminated with a loop. Relevant literature supporting the FMR measurement using coaxial probe is given in references [14 - 17].

The probe and sample at the angular frequency are modeled as a lumped-element circuit as shown in Fig. 1and the total impedance at the terminals of the equivalent transformer, $Z_{T}$, can be written as

$$
Z_{\text {Load }}(f) \approx i \omega L_{0}\left(1-k^{2}\right) \quad Z_{5}+(f) k^{2} \frac{L_{0}}{L_{x}}
$$

Where $L o$ is the probe/loop inductance, $Z_{S}$ is the complex surface impedance of the sample, $Z_{S}=R_{S}+i X_{S}, k$ is a dimensionless coefficient $(0 \leq k \leq 1)$ describing the probe to sample coupling,

and Lx is the inductance of the probe's image, and Lx is the inductance of the probe's image in the sample $\left(\mathrm{Lx} \longrightarrow L_{o}\right.$ for a perfect image). The total impedance is extracted from the reflection coefficient $S_{11}$ measured by a vector network analyzer (VNA) using the relation

$\mathrm{Z}_{\text {Load }}(\mathrm{f})=\left(1+\mathrm{S}_{11}\right) /\left(1-\mathrm{S}_{11}\right) \mathrm{Z}_{\mathrm{o}}$

After performing the in plane and out of plane FMR measurements, the $Z_{T}$ (In plane) and $Z_{T}$ (Out of plane) are extracted from $S_{11}$ equation shown above.

$\Delta Z_{\text {Load }}\left(f, H_{d c}\right)=Z_{\text {Load }}\left(f, H_{d c}^{\perp}\right)-Z_{\text {Load }}\left(f, H_{d c}^{\mathrm{p}}\right)=k^{2} \frac{L_{0}}{L_{X}}\left[R_{S}^{F M R}\left(f, H_{d c}\right)+i X_{S}^{F M R}\left(f, H_{d c}\right)\right]$

(3)

The permeability is extracted from the measurement using relations that follow

$$
\mu_{r} \approx k^{2} \frac{L_{X}}{L_{0}} \frac{1}{\omega t_{0} \mu_{0}}[\operatorname{Im}(\Delta Z)-i \operatorname{Re}(\Delta Z)]
$$

\subsection{HFSS Simulations on Ferrite Samples (Proof of Concept)}

The purpose of HFSS Simulations is to prove the concept of Ferromagnetic resonance measurement using short circuited probe. Spenato et al [18] proposed an analytic calculation of the complex permeability of thin amorphous ferromagnetic films, showing in-plane uniaxial anisotropy, subjected to an external in-plane alternating field perpendicular to the easy axis. The permeability tensor takes the form of following planar permeability equation. The effect of the parameters $M s, \mathrm{H}_{\mathrm{K}}$ and $\alpha$ on the complex permeability spectra is given below.

$$
\begin{aligned}
& \mu=\mu^{\prime}-\mu^{\prime \prime}=\left[\gamma_{\text {gyro }}^{2} 4 \pi M_{s}\left(H_{k}+4 \pi M_{s}\right)+j f \alpha 4 \pi M_{s}\right] \ldots \\
& \times\left[-f^{2}\left(1-\alpha^{2}\right)^{2}-f^{2} \alpha^{2}+\gamma_{\text {gyro }}^{2} H_{a}\left(H_{k}+4 \pi M_{s}\right) \quad j f \alpha \gamma_{g y r o}\left(2 H_{k}+4 \pi M_{s}\right)\right] \ldots \\
& \times\left\{\left[-f^{2}\left(1-\alpha^{2}\right)^{2}-f^{2} \alpha^{2}+\gamma_{\text {gyro }}^{2} H_{a}\left(H_{k}+4 \pi M_{s}\right) \quad \text { jf } \alpha \gamma_{\text {gyro }}\left(2 H_{k}+4 \pi M_{s}\right)\right]^{2} \ldots\right. \\
& \left.+f^{2} \alpha^{2} \gamma_{\text {gyro }}^{2}\left(H_{k}+4 \pi M_{s}\right)^{2}\right\}^{-1}+1
\end{aligned}
$$

Sligar et al [19] evaluated the initial permittivity and permeability of the ferrite samples (Lithium Ferrite, Magnesium Ferrite and Nickel Ferrite in the demagnetized state. The detailed description of theory and analysis are presented [19]. The broadband extraction of various important material characteristics of film magnetic materials is presented. The extracted complex permittivity and permeability for different demagnetized ferrite samples are found to be accurate within $2 \%$ of the input values. A detailed procedure to extract the complex permeability of the magnetized ferrite samples is also presented. The extracted values matched very closely with the behavior predicted by the theory of Stoner - Wohlfarth and derived permeability of Spenato which is shown in above equation. By utilizing the permeability values evaluated from the above equations, simulations were performed using coaxial probe on different ferrite samples with Inplane Magnetic Field and Out of Plane Magnetic Field to prove the concept. All these simulations were performed using Finite Element Method Tools HFSS (High Frequency Structure Simulator). The details of the coaxial probe are the Inner Conductor Diameter $=0.305 \mathrm{~mm}$; Outer Conductor Diameter $=2.655$ mm; Shorting Pin Length $=2.655$ mm; Shorting Pin Width $=0.1 \mathrm{~mm}$; Shorting Pin Thickness $=0.0005 \mathrm{~mm}$. The material specifications of the nickel ferrite used in the simulator are dielectric Constant $=13$; Dielectric Loss = 0.195; Magnetic Permeability $=300$, Saturation Magnetization=5000 Gauss; $\Delta \mathrm{H}=165$ Oe. Figure 1 shows the schematic of the coaxial probe. Figures 2 show the simulated results of the nickel ferrite sample along the Inplane and out of plane magnetic fields.

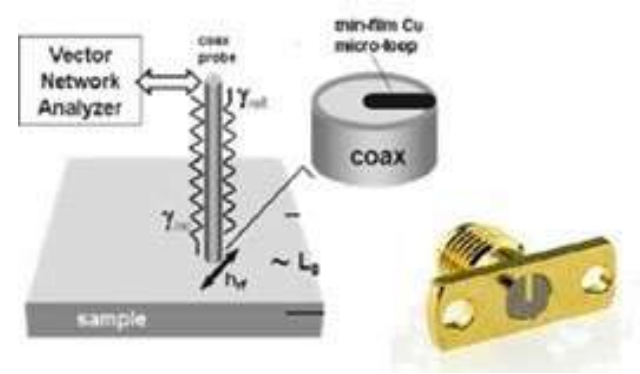

Figure 1: Schematic of the coaxial short circuited probe and equivalent circuit. 

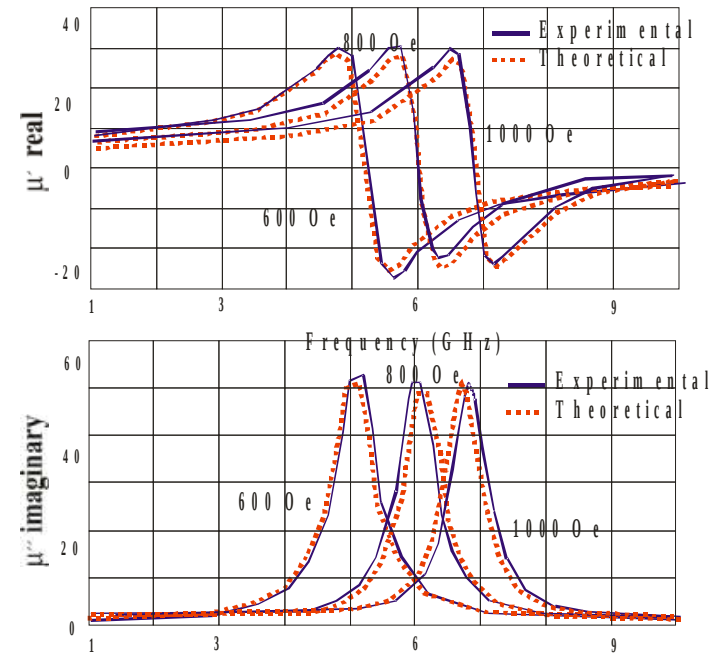

Erequency ( $G$ H $z$ )

(a)

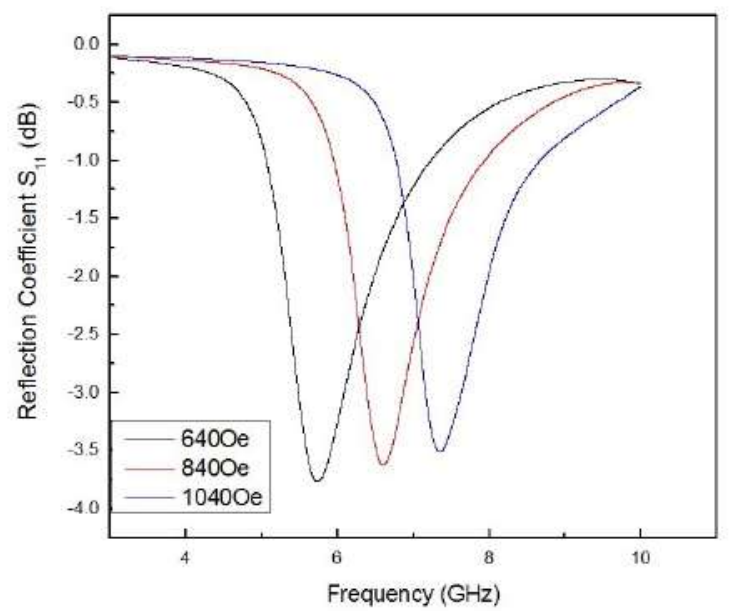

(b)

Figure 2: (a) show the simulated results of the nickel ferrite sample (NFO) along the in plane and out of plane magnetic fields. (b) show the theoretical and extracted values of the complex permeability for nickel ferrite sample.

\section{Results and Discussion}

The short-circuited loop probe is made of a coaxial cable with its inner conductor forming a loop shorted with the outer conductor. This design helps to enhance the magnetic coupling between the probe and sample, and further couples the microwave frequency currents of a controlled geometry in the sample. The probe consists of a SMA connector that transmits RF over $10 \mathrm{GHz}$. The dimensions of the probe are the outer diameter $(\mathrm{d} 1)$ of the conductor $=5.31 \mathrm{~mm}$ and inner diameter $(\mathrm{d} 2)$ of the conductor of the probe $=(0.6)$ $\mathrm{mm}$. The outer and inner conductor of the probe is shorted by a $\mathrm{Cu}-\mathrm{Pt}$ loop. $\mathrm{Cu}-\mathrm{Pt}$ loop is obtained by sputtering the copper and platinum films of thickness $500 \mathrm{~nm}$ and vary in width between $100 \mu \mathrm{m}$ and $500 \mu \mathrm{m}$ using the RF Magnetron
Sputtering unit. Masks of desired dimensions have been designed to have $\mathrm{Cu}-\mathrm{Pt}$ loops. In order to improve the sensitivity, the platinum film is deposited over copper film. The thin film probe design improves the sensitivity of the probe, minimizes the spacing between sample and probe resulting in enhanced electromagnetic coupling and the sheet film geometry generates a highly unidirectional RF field. The schematic of the short-circuited microwave coaxial probe for FMR studies over $1-8 \mathrm{GHz}$ is shown in Figure 1.

The experimental arrangement for the measurement of FMR consists of Microwave Vector Network Analyzer, SMA Shorted Coax Probe and DC Electromagnet. A standard calibration procedure for VNA Keysight N9918A was performed before measurements. A CW input signal with $\mathrm{f}$ $=2-8 \mathrm{GHz}$ and power Pin $=0.1 \mathrm{~mW}$ was applied to the short-circuited microwave coaxial probe. Low input power was chosen to prevent heating of the sample due to power absorption at FMR. Measurements are performed on both in plane and out of plane with applied magnetic fields (0 4000 Gauss) in the frequency range $(1-10 \mathrm{GHz})$.

The probe was then used for FMR studies on single crystal films of YIG with different thickness and polycrystalline samples of Nickel ferrite. Table 1 depicts the samples used for the microwave measurements. The film was grown by the liquid phase epitaxy on one side of a 0.5 -mm-thick gallium gadolinium garnet (GGG) substrate of <111> orientation. The film had a saturation magnetization of 1750 $\mathrm{G}$ and a FMR line width of $\sim 0.6 \mathrm{Oe}$, measured at $5 \mathrm{GHz}$. The YIG film was placed on the short circuited coaxial probe, so that the YIG film was in contact with the $\mathrm{Cu}-\mathrm{Pt}$ loop. Figure 8 presents the out of plane measured results on Yttrium Iron Garnet and Nickel ferrite Polycrystalline samples listed in Table 1. Profiles of reflected power Pref (f) vs. $\mathrm{f}$ were recorded for a series of H. Figure 2 depicts the measured FMR response of the YIG film with bias. The measured FMR linewidths of inplane and out of plane magnetic field for YIG and NFO samples are in the range of $0.4-3$ Oe.

Table 1: List of FMR sampler used for measurements

\begin{tabular}{|r|l|l|l|}
\hline & Sample & Thickness & Dimensions \\
\hline 1 & $\mathrm{YIG}\left(\mathrm{Y}_{3} \mathrm{Fe}_{5} \mathrm{O}_{12}\right)$ Single crystal film on GGG & $4.2 \mu \mathrm{m}$ & $2.0 \times 4 \mathrm{~mm}^{2}$ \\
\hline 2 & $\mathrm{YIG}\left(\mathrm{Y}_{3} \mathrm{Fe}_{5} \mathrm{O}_{12}\right)$ Single crystal film on GGG & $6.3 \mu \mathrm{m}$ & $2.0 \times 4 \mathrm{~mm}^{2}$ \\
\hline 3 & $\mathrm{YIG}\left(\mathrm{Y}_{3} \mathrm{Fe}_{5} \mathrm{O}_{12}\right)$ Single crystal film on GGG & $13.2 \mu \mathrm{m}$ & $2.0 \times 4 \mathrm{~mm}^{2}$ \\
\hline 4 & $\mathrm{YIG}\left(\mathrm{Y}_{3} \mathrm{Fe}_{5} \mathrm{O}_{12}\right)$ Single crystal film on GGG & $24 \mu \mathrm{m}$ & $2.0 \times 4 \mathrm{~mm}^{2}$ \\
\hline 5 & $\mathrm{YIG}\left(\mathrm{Y}_{3} \mathrm{Fe}_{5} \mathrm{O}_{12}\right)$ Single crystal film on GGG & $100 \mu \mathrm{m}$ & $2.0 \times 4 \mathrm{~mm}^{2}$ \\
\hline 6 & $\mathrm{YIG}\left(\mathrm{Y}_{3} \mathrm{Fe}_{5} \mathrm{O}_{12}\right)$ Single crystal film on GGG & $124 \mu \mathrm{m}$ & $2.0 \times 4 \mathrm{~mm}^{2}$ \\
\hline 7 & $\mathrm{YIG}\left(\mathrm{Y}_{3} \mathrm{Fe}_{5} \mathrm{O}_{12}\right)$ Polycrystalline & $0.5 \mathrm{~mm}$ & $2.0 \times 4 \mathrm{~mm}^{2}$ \\
\hline 8 & Nickel $\left.\mathrm{Ferrite}_{(\mathrm{NiFe}} \mathrm{O}_{4}\right)$ Polycrystalline & $0.5 \mathrm{~mm}$ & $2.5 \times 4 \mathrm{~mm}^{2}$ \\
\hline
\end{tabular}




\section{Conclusions}

A compact measurement methodology for the measurement of Ferromagnetic resonance using Short Circuit Coax Probe Method over a wide frequency range has been presented. A detailed theoretical approach and simulation studies using HFSS have been performed. Microwave measurements (Ferromagnetic Resonance) were carried out using vector network analyzer, shorted coaxial probe and DC electromagnet on the different ferrite samples (single crystal YIG films with different thickness and polycrystalline Nickel Ferrite). Both Inplane and out of plane measurements are performed and respective resonant line widths on these samples are calculated in the frequency range of $1-10 \mathrm{GHz}$.

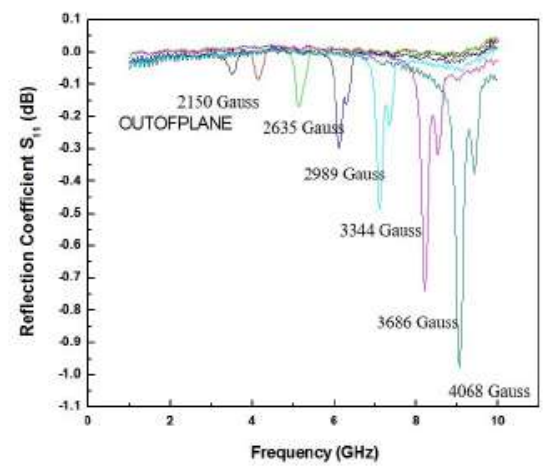

(a)

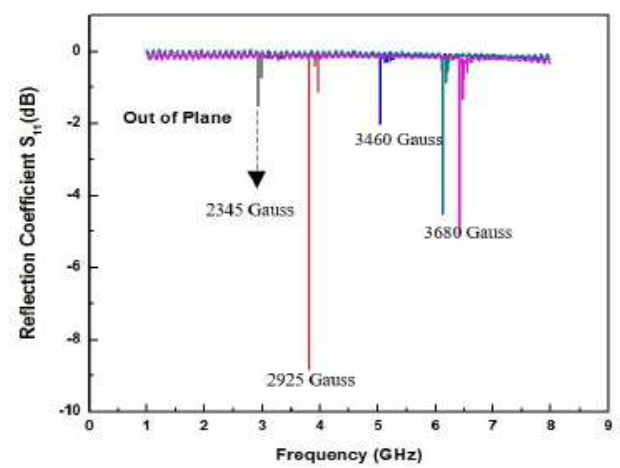

(b)

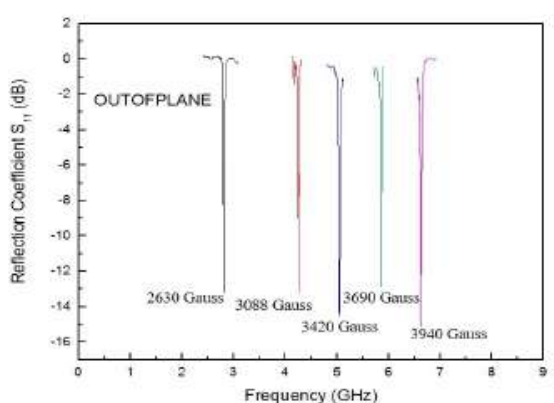

(c)

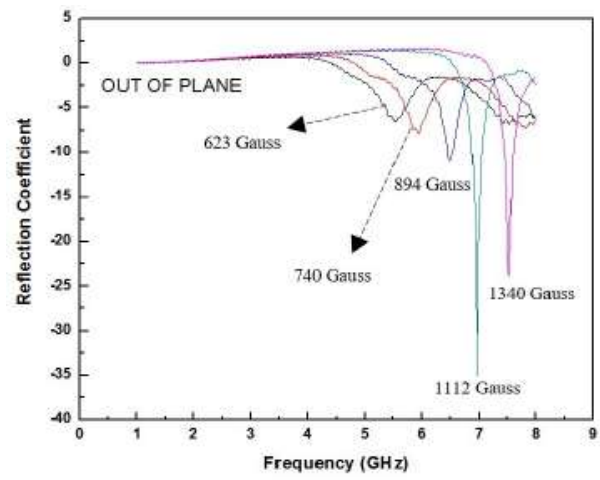

(d)

Figure 3: Ferromagnetic Resonance (FMR) measurements using shorted coaxial probe method on ferrite materials a) YIG Single Crystal film (thickness 4 $\mu \mathrm{m}) \mathrm{b}$ ) YIG Single Crystal film (thickness $24 \mu \mathrm{m}) \mathrm{c}$ ) YIG Single Crystal film (thickness $124 \mu \mathrm{m}$ ) d) NFO Polycrystalline sample (thickness $0.2 \mathrm{~mm}$ ).

\section{Acknowledgements}

The authors would like to acknowledge Intel Corporation for the financial support which made this work possible 


\section{References}

[1] B. Kuanr, R. E. Camley, and Z. Celinski., Narrowing of the frequency-linewidth in structured magnetic strips: Experiment and theory., Applied Physics Letters, 87, 012502 (2005).

[2] V. Korenivski, R. B. van Dover, P. M. Mankiewich, Z.X. Ma, A. J.Becker, P. A. Polakos, and V. J. Fratello, A method to measure the complex permeability of thin films at ultra-high frequencies, IEEE Trans. Magn.,vol.32, no.5, pp. 4905 - 4907 (1996).

[3] Y. Ding, T. J. Klemmer, and T. M. Crawford, A coplanar waveguide permeameter for studying high-frequency properties of soft magnetic materials, J. Appl. Phys., vol.96, pp. 2969, (2004).

[4] K R Smith, M J Kabatek, P Krivosik, M Wu, "Spin wave propagation in spatially nonuniform magnetic fields", J. Appl. Phys., vol. 104, 043911, 2008.

[5] D. Rugar, R. Budakian, H. J. Mamin, and B. W Chui, Single spin detection by magnetic resonance force microscopy,Nature, 2004, 430: 329.

[6] R. Meckenstock, Microwave spectroscopy based on scanning thermal microscopy: resolution in the nanometer range.,Rev. Sci. Instrum., 2008, 79: 041101

[7] Y. Obukhov, D. V. Pelekhov, J. Kim, P. Banerjee, I. Martin, E. Nazaretski, R. Movshovich, S. An, T. J. Gramila, S. Batra, and P. C. Hammel, Local ferromagnetic resonance imaging with magnetic resonance force microscopy, Phys. Rev. Lett., 2008, 100: 197601

[8] T. An, N. Ohnishi, T. Eguchi, Y. Hasegawa, and P. Kabos," "Local excitation of ferromagnetic resonance and its spatially resolved detection with an open-ended radio-frequency probe,” IEEE Magn. Lett., 2010, 1: 3500104

[9] S. C. Lee, C. P. Vlahacos, B. J. Feenstra, A. S. Schwartz, D. E. Steinhauer, F. C. Wellstood, and S. M. Anlage, Magnetic permeability imaging of metals with a scanning near-field microwave microscope,Appl. Phys. Lett., 2000, 77: 4404

[10] D. I. Mircea and T. W. Clinton, Near-field microwave probe for local ferromagnetic resonance characterization, Appl. Phys. Lett., 2007, 90: 142504

[11] Y. K. Fetisov and G. Srinivasan, "Electric field tuning characteristics of a ferrite-piezoelectric microwave resonator”, Appl. Phys. Lett., 2006, 88, 143: 503

[12] A. B. Ustinov, Yu. K. Fetisov, and G. Srinivasan, "Electric switching in bistable ferrite-piezoelectric microwave resonator” Tech. Phys. Lett., 2008, 34: 593

[13] N. Benatmane, S. P. Crane, F. Zavaliche, R. Ramesh, and T. W. Clinton, "Voltage-dependent ferromagnetic resonance in epitaxial multiferroic nanocomposites”, Appl. Phys. Lett., 2010, 96: 082503

[14] Dragos I. Mircea and T.W. Clinton., Near field Microwave Probe for Local ferromagnetic resonance Characterization. Applied Physics Letters 90, 142504 (2007).
[15] T.W.Clinton, Dragos I. Mircea, Nadjib Benatmane, N.J.Gokemeijer, Stella Wu, and S.D.Harkness IV., A Nondestructive Local FMR Probe for On-Disk Media Characterization. IEEE Transactions on Magnetics. 43, no.6, pp.2319 -2321 (2007)

[16] Toshu An Nobuhito Ohnishi, Toyoaki Eguchi, Yukio Hasegawa, and Pavel Kabos., Local Excitation of Ferromagnetic Resonance and its Spatially Resolved Detection with an Open Ended Radio - Frequency Probe. IEEE Magnetics Letters, 1, 3500104 (2010)

[17] Mahmoud Nadjib Benatmane, (Ph.D., Dissertation) Development and Application of a Novel Near Field Microwave Probe for Local Broadband Characterization of Ferromagnetic Resonance. Febrauary (2010)

[18] D. Spenato, A Fessant, J Gieraltowski, J Loaec and H Le Gall., Theoretical and experimental approach of spin dynamics in in-plane anisotropic amorphous ferromagnetic thin films. J. Phys. D: Appl. Phys., Vol. 26, pp.1736 - 1740, (1993).

[19] Arien Sligar, (Ph.D., Dissertation) On - Chip Crosstalk Suppression Schemes using Magnetic Films for RF/Microwave Applications. June (2007) 